\title{
A Stress-Strain Framework for Modelling the Behaviour of Unsaturated Soils under Non-Isothermal Conditions
}

\author{
B. François and L. Laloui
}

Soil Mechanics Laboratory, Ecole Polytechnique Fédérale de Lausanne, Station 18, CH-1015 Lausanne, Switzerland

bertrand.francois@epfl.ch, lyesse.laloui@epfl.ch

Summary. This paper addresses a new, unified thermo-mechanical constitutive model for unsaturated soils through a coupled study. In the context of elastoplasticity and the critical state theory, the model uses the concepts of multimechanism and bounding surface theory. This advanced constitutive approach involves thermo-plasticity of saturated and unsaturated soils. Bishop's effective stress framework is adopted to represent the stress state in the soil. This stress is linked to the water retention curve, which is represented by an elasto-plastic model. Attention is focused particularly on the coupling relations inferred from this unified thermohydro-mechanical (THM) study. Finally, the theoretical aspects of the paper are supported by comparisons between numerical simulations and experimental results.

Key words: unsaturated soils, thermo-mechanics, constitutive modelling, elastoplasticity, multi-mechanism

\section{Introduction}

Research interest in the thermo-mechanical behaviour of unsaturated soils is growing as a result of an increasing number of geomechanical problems involving both thermal and capillary effects. In the field of environmental geomechanics, several relevant applications, such as feasibility studies of waste disposal, petroleum extraction or geothermal structures, require an accurate knowledge of the thermo-mechanical behaviour of unsaturated soils (Vulliet et al., 2002). Until now, these two loading cases have often been considered independently.

This paper addresses a new, unified thermo-mechanical constitutive model for unsaturated soils, named ACMEG-TS ("Advanced Constitutive Model in Environmental Geomechanics - Thermal and Suction effects"). This constitutive model is based on thermo-hydro-mechanical (THM) experimental data and attention is focused on the coupling relations linking the thermal, hydric 
and mechanical soil response. After presenting the stress framework used here, the different experimental data for THM soil behaviour are addressed. The constitutive equations are then introduced. Finally, ACMEG-TS is validated for several typical THM paths.

\section{Stress Framework}

An unsaturated state within the soil means that a second fluid phase appears in the inter-particular spaces. The difference in pressure between these two phases (air and water) leads to a new stress variable, suction: $s=u_{a}-u_{w}$ which modifies the internal stress state in the soil. Bishop's effective stress is very useful for continuously describing the three phase nature of unsaturated soils (Bishop, 1959; Khalili et al., 2005):

$$
\sigma_{i j}^{\prime}=\left(\sigma_{i j}-u_{a} \delta_{i j}\right)+\chi\left(u_{a}-u_{w}\right) \delta_{i j}
$$

where $\sigma_{i j}$ is the total external stress, $u_{a}$ and $u_{w}$ are the air and water pore pressures, respectively, $\chi$ the effective stress parameter and $\delta_{i j}$ the Kroenecker's symbol. ACMEG-TS uses the generalised Bishop's effective stress, where $\chi$ is equal to the degree of saturation $S_{r}$, as suggested in the initial work of Bishop (1959) and implemented by Schrefler (1984). Equation (1) requires hydric information (represented by $S_{r}$ ) in parallel with the stress state within the soil. The degree of saturation is related to suction through the retention curve. It is shown that this $S_{r}$ versus $s$ curve depends on temperature (Salager et al., 2006), on the dry density of the soils (Salager et al., 2007) and on the hydric paths followed (wetting or drying). Thus, the effective stress depends on the thermal, hydric and mechanical histories of the material. Therefore, this single stress approach converts a complex, multi-phase and multi-stress medium in which multi-physics processes occur into a single mechanical state through several coupling equations.

\section{THM Experimental Data}

Under normally consolidated conditions (NC), clayey soil contracts when it is heated and a significant part of this deformation is irreversible upon cooling (Laloui, 2001). This behaviour over the whole cycle is representative of thermal hardening. Another important non-isothermal behaviour is the fact that the apparent preconsolidation pressure decreases with increasing temperature while the isotropic compressibilities do not seem to be significantly affected by temperature changes (Laloui and Cekerevac, 2003). In addition to these thermal effects on the isotropic soil response, hydric conditions partially govern the mechanical response of the soil. Indeed, it is shown that the apparent preconsolidation pressure increases when suction increases for suctions greater 
than the air entry suction, $s_{e 0}$, and is uninfluenced by suctions lower than $s_{e 0}$ (Geiser et al., 2006).

The thermal, as well as the capillary, effects on soil strength seem to be negligible. Cekerevac and Laloui (2004) summarized some experimental results which tend to confirm that the friction angle at critical state can either slightly increase or decrease with temperature. Moreover, in the generalized Bishop's effective stress reference, the friction angle appears to be independent of the amount of suction (Khalili et al., 2004).

\section{ACMEG-TS Constitutive Framework}

\section{Mechanical Framework}

The basic concept of the ACMEG-TS model is to consider that the thermal, as well as the hydric, loads exclusively involve volumetric effects in soils. These considerations are introduced in an (THM) elasto-plastic framework where each THM loading (external mechanical load, $\sigma$, temperature, $T$, and suction, $s$ ) may imply reversible and irreversible changes in the state of the material. Within this elasto-plastic framework, the total strain rate tensor, $\dot{\varepsilon}$, due to THM loading is decomposed into non-linear, thermo-elastic, $\dot{\varepsilon}^{e}$, and thermo-plastic, $\dot{\varepsilon}^{p}$, components. Due to the isotropic effect of the temperature and the hydric changes in the soil, the plastic mechanism of the material is induced by two coupled hardening processes: an isotropic one which may be activated by any mechanical, thermal or hydric loads and a deviatoric mechanism acting only under a mechanical loading having a deviatoric component. Both mechanisms may induce volumetric plastic strain (Laloui et al., 2005). Therefore the total volumetric plastic strain rate $\dot{\varepsilon}_{v}^{p}$ is the coupling variable linking the two hardening processes and is given by:

$$
\dot{\varepsilon}_{v}^{p}=\dot{\varepsilon}_{v, \text { iso }}^{p}+\dot{\varepsilon}_{v, \mathrm{dev}}^{p}
$$

where $\dot{\varepsilon}_{v, \text { iso }}^{p}$ and $\dot{\varepsilon}_{v \text {,dev }}^{p}$ are the volumetric plastic strain rate induced by the isotropic and the deviatoric mechanisms, respectively. The yield functions of the two mechanical, thermo-plastic mechanisms have the following expressions (Fig. 1):

$$
f_{\text {iso }}=p^{\prime}-\sigma_{c}^{\prime} r_{\text {iso }}=0 ; \quad f_{\text {dev }}=q-M p^{\prime}\left(1-b \log \frac{d p^{\prime}}{\sigma_{c}^{\prime}}\right) r_{\mathrm{dev}}=0
$$

where the preconsolidation pressure, $\sigma_{c}^{\prime}$, depends on the volumetric plastic strain, $\varepsilon_{v}^{p}$, temperature, $T$, and suction, $s$ (Laloui and Nuth, 2005):

$$
\sigma_{c}^{\prime}= \begin{cases}\sigma_{c 0}^{\prime} \exp \left(\beta \varepsilon_{v}^{p}\right)\left\{1-\gamma_{T} \log \left(\frac{T}{T_{0}}\right)+\gamma_{s} \log \left(\frac{s}{s_{e 0}}\right)\right\} & \text { if } s>s_{e 0}, \\ \sigma_{c 0}^{\prime} \exp \left(\beta \varepsilon_{v}^{p}\right)\left\{1-\gamma_{T} \log \left(\frac{T}{T_{0}}\right)\right\} & \text { if } s \leq s_{e 0} .\end{cases}
$$




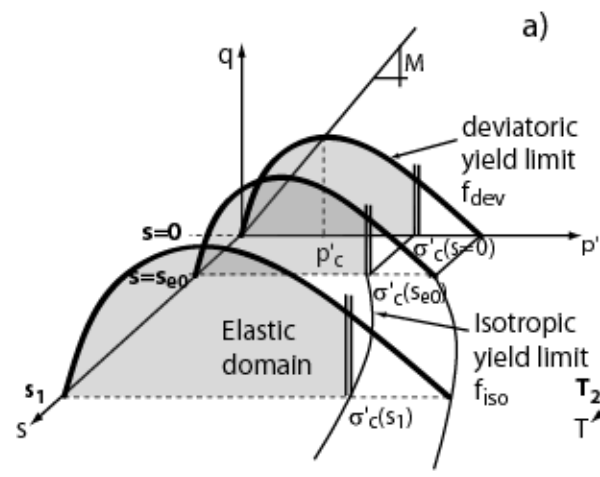

a)

b)

Fig. 1. Hydric (a) and thermal (b) effects on the THM yield limits

$r_{\text {iso }}$ and $r_{\text {dev }}$ are the degree of mobilization of the isotropic and the deviatoric mechanisms and are hyperbolic functions of the plastic volumetric strain induced by the isotropic and the deviatoric mechanisms, respectively. They vary from $r^{e}$, which defines the elastic domain $\left(0<r^{e}<1\right)$, to 1 at a completely plastic state (Hujeux, 1979).

$\gamma_{T}$ and $\gamma_{s}$ are the material parameters required to quantify the effect of temperature and suction, respectively, on the apparent preconsolidation pressure. $\beta$ is the plastic compressibility modulus (the slope of the linear function $\left.\varepsilon_{v}^{p}-\log \sigma_{c}^{\prime}\right) . s_{e 0}$ is the air entry suction which depends on the temperature and plastic volumetric strain, as expressed in equation (6).

\section{Hydric Framework}

In terms of hydric response, the desaturation process is also seen as a yielding phenomenon. As long as the soil is drying, suction increases and the degree of saturation, $S_{r}$, tends to decrease mainly when the air entry suction, $s_{e 0}$, is reached. In this way, $s_{e 0}$ can be seen as a hydric yield limit with the following yield function:

$$
f_{\text {hyd }}=s-s_{e}=0
$$

where the hydric limit, $s_{e}$, depends on the degree of saturation, the volumetric plastic strain and the temperature (Fig. 2):

$$
s_{e}=s_{e 0} \exp \left(-\beta_{h} \Delta S_{r}\right)\left\{1-\theta_{T} \log \left(\frac{T}{T_{0}}\right)-\theta_{e} \log \left(1-\varepsilon_{v}^{p}\right)\right\} .
$$

$\theta_{T}$ and $\theta_{e}$ are the material parameters required to quantify the effect of temperature and volumetric plastic strain on the evolution of the air entry suction, respectively. $\beta_{h}$ is the slope of the linear function $S_{r}-\log s$ when the air entry suction is overcome. For the wetting process, the same elasto-plastic concept can also be applied by considering the hydric hysteresis through an additional material parameter, $s_{\text {hys }}$, quantifying the hydric irreversibility (Fig. 2). 


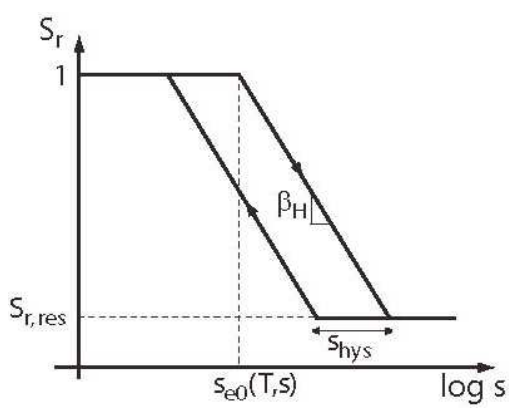

Fig. 2. The air entry suction as the key parameter in the water retention curve

\section{Numerical Simulations}

A set of oedometric compression tests were carried on a remoulded sandy silt at different temperatures and suctions (François et al., 2007). The results of these experiments clearly show the thermal and hydric effects on the apparent preconsolidation pressure. The comparison between numerical simulations and experimental results show the ability of ACMEG-TS to represent such THM coupling (Fig. 3). For this simulation, the three key parameters are $\left\{\gamma_{T} ; \gamma_{s} ; \beta\right\}=\{0.8 ; 0.28 ; 37\}$. Moreover, the model is able to reproduce the typical thermal hardening observed along a temperature cycle (Baldi et al., 1991) (Fig. 4).

\section{Conclusions}

A highly-coupled THM model (ACMEG-TS) has been presented. It is based on two interrelated constitutive schemes, the mechanical and the hydric one.

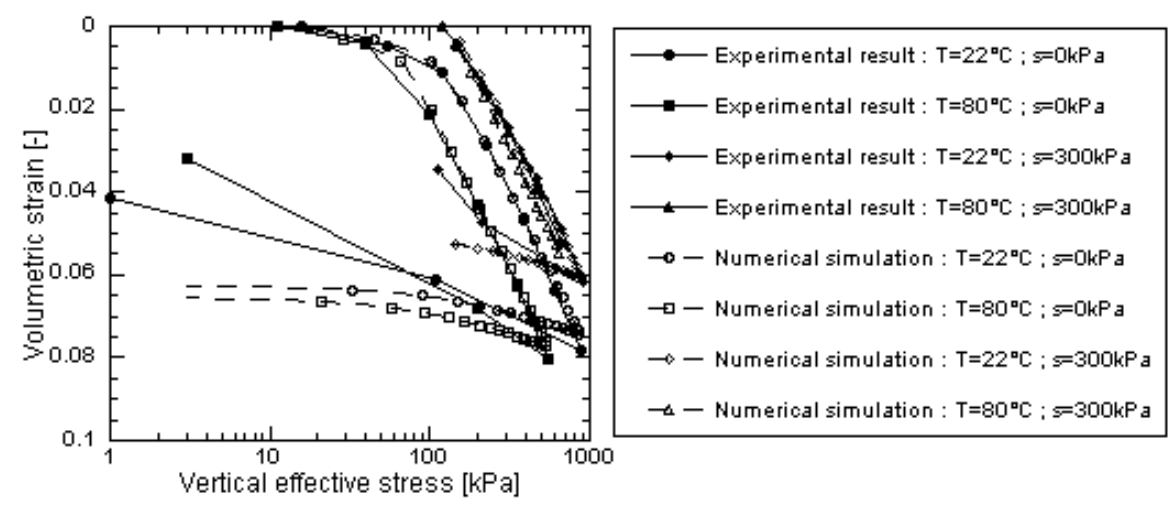

Fig. 3. Numerical simulations of THM oedometric compression tests on a sandy silt 


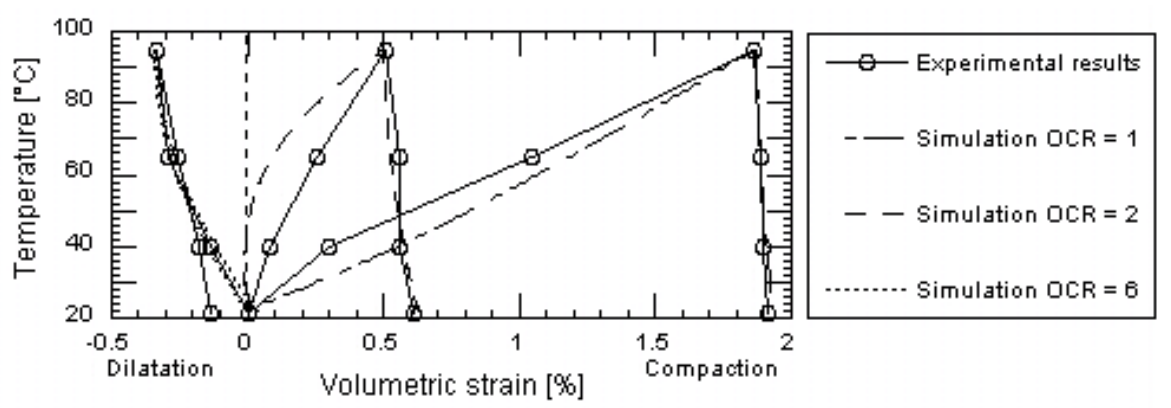

Fig. 4. Numerical simulations of the mechanical response of Boom Clay along a thermal cycle

The hydric condition influences the isotropic mechanical behaviour via its effect on the apparent preconsolidation pressure. Inversely, the mechanical state may have an effect on the hydric response by modifying the water retention curve (via the evolution of the air entry suction). Moreover, these two-way couplings depend on temperature. Therefore, this THM constitutive model considers the three loading cases (external load, suction and temperature) as three interconnected phenomena.

\section{Acknowledgements}

This work was partly funded by the Swiss State Secretariat for Education and Research SER, Grant OFES C04.0021.

\section{References}

Baldi G, Hueckel T, Peano A, Pellegrini R (1991) Developments in modelling of thermo-hydro-mechanical behaviour of Boom clay and clay-based buffer materials (Vol 1 and 2). EUR 13365/1 and 13365/2, Luxembourg

Bishop AW (1959) The principle of effective stress, Teknisk Ukeblad 39:859-863

Cekerevac C, Laloui L (2004) Experimental study of thermal effects on the mechanical behaviour of clays, Int J Num Anal Meth in Geomechanics 28:209-228

François B, Salager S, El Youssoufi MS, Ubals Picanyol D, Laloui L, Saix C (2007) Compression tests on a sandy silt at different suction and temperature level. In: GeoDenver07 ASCE Conference (Submitted)

Geiser F, Laloui L, Vulliet L (2006) Elasto-plasticity of unsaturated soils: laboratory test results on a remoulded silt, Soils and Foundations Journal 46(5) (in press)

Hujeux J (1979) Calcul numérique de problèmes de consolidation élastoplastique. PhD Thesis, Ecole Centrale Paris

Khalili N, Witt R, Laloui L, Vulliet L, Koliji A (2005) Effective stress in double porous media with two immiscible fluids, Geophysical Research Letters 32(15):L15309 
Khalili N, Geiser F, Blight GE (2004) Effective Stress in Unsaturated Soils: Review with New Evidence, Int J Geomech 4(2):115-126

Laloui L (2001) Thermo-mechanical behaviour of soils, Revue Française de Génie Civil 5:809-843

Laloui L, Cekerevac C (2003) Thermo-plasticity of clays: an isotropic yield mechanism, Computers and Geotechnics 30:649-660

Laloui L, Cekerevac C, François B (2005) Constitutive modelling of the thermoplastic behaviour of soils, Revue Européenne de Génie Civil 9:635-650

Laloui L, Nuth M (2005) An introduction to the constitutive modelling of unsaturated soils, Revue Européenne de Génie Civil 9(5-6):651-670

Salager S, El Youssoufi MS, Saix C (2007) Experimental study of the water retention curve as a function of void ratio. In: GeoDenver07 ASCE Conference (Submitted)

Salager S, Jamin F, El Youssoufi MS, Saix C (2006) Influence de la température sur la courbe de rétention d'eau de milieux poreux. C. R. Mécanique 334:393-398

Schrefler BA (1984) The finite element method in soil consolidation (with applications to surface subsidence). Ph.D. Thesis, University College of Swansea, $\mathrm{C} / \mathrm{Ph} / 76 / 84$

Vulliet L, Laloui L, Schrefler BA (2002) Environmental Geomechanics. EPFL-Press, $423 \mathrm{pp}$ 